\title{
A Remark on Relevant ARithmetic
}

\author{
Chris Mortensen
}

University of Adelaide

I recall reading Bob Meyer's abstract "Relevant Arithmetic" [1] for the first time around 1980. I was immediately struck by what seemed to be an error. On page 133, after setting up finite inconsistent arithmetic which we later called RM3 $\bmod 2$, and observing that this provided a finitary proof that $0=1$ was not a theorem of its subtheory relevant arithmetic $R^{\sharp}$, he wrote:

Similarly, every other incorrect quantifier equation (which may contain variables) may be refuted... $\mathrm{R}^{\sharp}$ is arithmetically consistent in the sense that equations which violate the addition or multiplication tables for + and $\times$ are trivially undemonstrable.[1, p. 133-4]

How could this be? I agreed that it can be proved by finitary means that $0=1$ fails in RM3 mod 2 and so $R^{\sharp}$. But even as simple an equation as $0=2$ holds in RM3 mod 2 and so provides no reason to conclude that it does not hold in $R^{\sharp}$, that is, that $R^{\sharp}$ is arithmetically consistent.

I stared at this for several days, frustrated. Then the penny dropped, with a huge clang. To refute $0=2$, take $R M 3 \bmod 3$, in which $0=1$ and $0=2$ fail but $0=3$ holds. By a similar finitary argument $R^{\sharp}$ is a subtheory of $R M 3 \bmod 3$, so since $0=2$ fails in $R M 3 \bmod 3$, it fails in $R^{\sharp}$.

All of a sudden a vast collection of arithmetical theories fall into place. To refute in $\mathrm{R}^{\sharp}$ any false arithmetical equation, say $3+6=4 \times 4$, calculate each side out, $9=16$, then take the least mod greater than both sides, mod 17 , form RM3 mod 17 , in which $9=16$ fails, show by induction that $R^{\sharp}$ is a subtheory, and we are done.

Moreover, Bob must have seen this or he wouldn't have said the above. When I taxed him several days later, he just laughed.

Australasian Journal of Logic (18:5) 2021, Article no. 8 
It is also obvious that this array of theories has properties of its own, intersect them, union them, take lcms, hcfs, prime mods, nonstandard extensions, and all that. Obviously, this leads straight to the models of "Inconsistent Models for Relevant Arithmetics" [2].

\section{References}

[1] R. K. Meyer. Relevant arithmetic. Bulletin of the Section of Logic, 5:133-137, 1976.

[2] R. K. Meyer and C. Mortensen. Inconsistent models for relevant arithmetics. Journal of Symbolic Logic, 49(3):917-929, 1984.

Australasian Journal of Logic (18:5) 2021, Article no. 8 